\title{
Aspectos anatômicos e clínicos da síndrome braquicefálica: revisão de literatura
}

\author{
Anatomical and clinical aspects of brachycephalic syndrome: literature review \\ Aspectos anatómicos y clínicos del síndrome braquicefálico: revisión de la literatura
}

Recebido: 29/09/2021 | Revisado: 04/10/2021 | Aceito: 09/10/2021 | Publicado: 11/10/2021

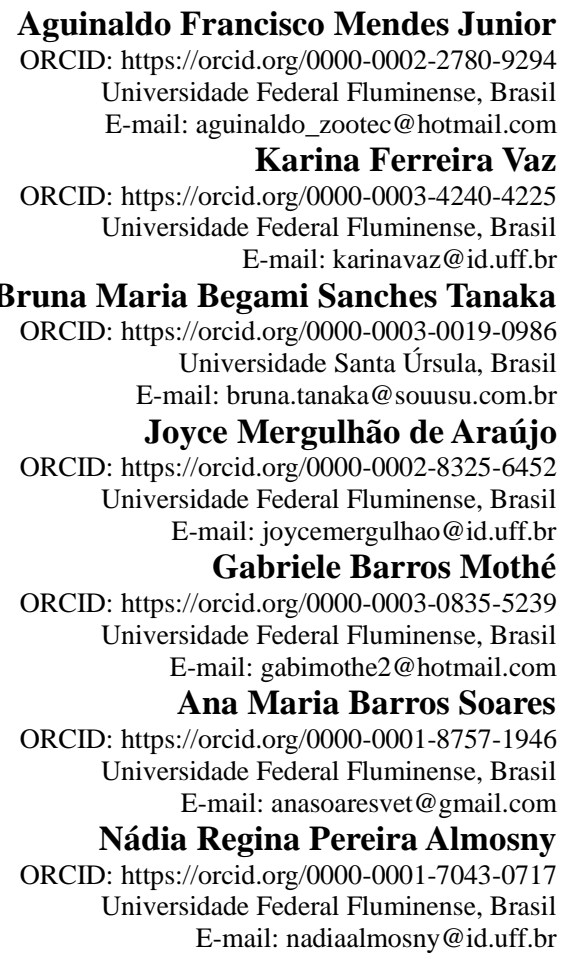

\section{Resumo}

A grande popularidade dos cães braquicefálicos entre os tutores levou à necessidade de um maior conhecimento por parte dos médicos veterinários das doenças características dessas raças. Chamam a atenção, principalmente, as modificações relacionadas às vias aéreas anteriores, que sofreram grandes alterações e predispõem ao desenvolvimento de uma grave doença multissistêmica conhecida como síndrome dos cães braquicefálicos (SB) ou síndrome respiratória obstrutiva dos braquicéfalos (SROB). O estudo ora conduzido tem por objetivo apresentar uma revisão evidenciando os aspectos clínicos e anatômicos da SB visto a importância desta doença na clínica médica e cirúrgica de pequenos animais. Podendo desta forma concluir que o reconhecimento precoce das alterações anatômicas obstrutivas que caracterizam a síndrome braquicefálica pelo médico veterinário é de extrema importância para a pronta instituição do tratamento possibilitando melhores resultados e assim melhor qualidade de vida para o animal.

Palavras-chave: Estenose de narinas; Prolongamento de palato mole; Obstrução de vias aéreas.

\begin{abstract}
The great popularity of brachycephalic dogs among tutors led to the need for greater knowledge on the part of veterinarians about the characteristic diseases of these breeds. Particularly noteworthy are the modifications related to the anterior airways, which have undergone major changes and predispose to the development of a severe multisystem disease known as brachycephalic dog syndrome (SB) or obstructive respiratory syndrome of the brachycephalic (BOAS). The present study aims to present a literature review highlighting the clinical and anatomical aspects of BS considering the importance of this disease in the medical and surgical clinic of small animals. Thus, it can be concluded that the early recognition of the obstructive anatomical changes that characterize the brachycephalic syndrome by the veterinarian is extremely important for the prompt institution of the treatment, enabling better results and thus better quality of life for the animal.
\end{abstract}

Keywords: Nostril stenosis; Soft palate extension; Airway obstruction. 


\begin{abstract}
Resumen
La gran popularidad de los perros braquicefálicos entre los tutores llevó a la necesidad de un mayor conocimiento por parte de los veterinarios sobre las enfermedades características de estas razas. Destacan las modificaciones relacionadas con las vías aéreas anteriores, que han sufrido cambios importantes y predisponen al desarrollo de una enfermedad multisistémica grave conocida como síndrome del perro braquicefálico (SB) o síndrome respiratorio obstructivo del braquicéfalo (BRS). El presente estudio tiene como objetivo presentar una revisión de la literatura destacando los aspectos clínicos y anatómicos del SB considerando la importancia de esta enfermedad en la clínica médica y quirúrgica de pequeños animales. Así, se puede concluir que el reconocimiento precoz de los cambios anatómicos obstructivos que caracterizan al síndrome braquicéfalo por parte del veterinario es de suma importancia para la pronta institución del tratamiento, permitiendo mejores resultados y por tanto una mejor calidad de vida del animal.
\end{abstract}

Palabras clave: Estenosis de las fosas nasales; Prolongación del paladar blando; Obstrucción de la vía aérea.

\title{
1. Introdução
}

A grande popularidade dos cães braquicefálicos entre os tutores levou à necessidade de um maior conhecimento das doenças características dessas raças (Meola, 2013). Chamam a atenção, principalmente, as modificações relacionadas às vias aéreas anteriores, que sofreram grandes alterações. Essas alterações predispõem ao desenvolvimento de uma grave doença respiratória conhecida como síndrome dos cães braquicefálicos (SB) ou síndrome respiratória dos braquicéfalos (SROB) (Packer; Hendricks; Tivers \& Burn, 2015).

A síndrome respiratória dos braquicéfalos pode ser observada em cães e gatos de focinho curto. É constituída por alterações anatômicas que causam importante obstrução das vias aéreas anteriores, impedindo o fluxo adequado do ar até os pulmões. Inúmeras deformações obstrutivas de etiologia congênita compõem a SB, como estenose bilateral de narinas, presença de cornetos nasais aberrantes, macroglossia, redução dos espaços naso e orofaríngeo, diminuição do espaço retrofaríngeo, prolongamento de palato mole e hipoplasia de traqueia. Em função do caráter progressivo da doença, esses animais podem apresentar alterações secundárias, como espessamento de palato mole, edema e inflamação de orofaringe e nasofaringe, cistos epiglóticos, hiperplasia de tonsilas, sialocele, granulomas de laringe, eversão dos sáculos laríngeos, paralisia ou colapso de laringe, colapso de traqueia e colapso de brônquio principal, que podem agravar o estado clínico do paciente (Emmerson, 2014; Dupré \& Heidenrich, 2016).

Sinais clínicos significativos são observados em função da fisiopatologia da doença, entre os quais ronco, estertor, estridor, dispneia, cianose de mucosa, perturbações do sono, intolerância ao exercício, dificuldade de recuperação após os exercícios, alterações gastrointestinais e cardíacas, intermação e edema pulmonar pós-obstrutivo são comumente encontrados. Esses sinais apresentam grande potencial deletério para a qualidade de vida dos animais e, em muitos casos, alto risco de óbito (Emmerson, 2014; Packer \& Tivers, 2015; Dupré \& Heidenrich, 2016). Dessa forma, torna-se extremamente importante o conhecimento das características anatômicas e clínicas da síndrome braquicefálica pelos médicos veterinários para correta identificação das alterações obstrutivas para melhor diagnóstico e tratamento da doença.

\section{Metodologia}

Foi realizada revisão de literatura narrativa (Pereira, Shitsuka, Parreira \& Shitsuka 2018) incluindo artigos científicos publicados e disponíveis nas bases de dados: Capes (Coordenação de Aperfeiçoamento de Pessoal de Nível Superior), Scielo (Scientific Electronic Library Online), Sistema Latino Americano e do Caribe de Informação em Ciências da Saúde (LILACS), PUBMED e Google acadêmico.

Para critérios de inclusão, foram utilizadas 69 publicações com datas desde 1997 até 2021, nas línguas portuguesa e inglesa, com títulos e resumos relacionados à síndrome braquicefálica utilizando os seguintes descritores: síndrome braquicefálica, estenose de narinas e prolongamento de palato mole. 
Foram excluídos artigos que não apresentavam o resumo, e não abordavam a temática em estudo, bem como artigos de opinião que não estavam apoiados em dados de pesquisa científica ou que não apresentavam suporte de uma coleta sistemática de dados.

Os dados foram coletados através da leitura inicial dos artigos e livros selecionados, sendo em seguida destacadas as informações de maior interesse em cada artigo publicado. Posteriormente foi feita a leitura na íntegra dos artigos referentes ao tema proposto, obtendo-se um conjunto de informações, na qual foram relacionadas com o objetivo, permitindo o desenvolvimento do atual estudo.

\section{Revisão de Literatura}

Ao longo de dezenas de milhares de anos, com o objeto de adequação aos padrões de cada raça, o ser humano submeteu os cães a intensos processos de melhoramento genético. A atenção dispensada à seleção de exemplares com características fenotípicas específicas consideradas desejáveis em cada raça levou ao surgimento de variações na conformação craniana dos animais. Essas medidas intensificaram o desenvolvimento de importantes doenças, muitas vezes relacionadas ao formato do crânio (Schoenebeck \& Ostrander, 2013).

A seleção de cães com o formato do crânio mais achatado leva a uma aparência infantilizada do animal, garantindo, assim, maior aceitação dos proprietários. Porém, torna-se um fator de risco para a ocorrência da síndrome respiratória do cão braquicefálico (Packer et al., 2015).

Ao estudar o processo de fechamento das suturas cranianas em cães braquicefálicos e mesaticefálicos, por meio de ressonância magnética, Schmidt, Amort, Failing, Klingler, Kramer e Ondreka (2013), concluíram que os braquicéfalos apresentam um fechamento mais precoce das sincondroses esfeno-occipitais. Os autores relataram que esta pode ser considerada uma das possíveis causas da conformação craniana característica dos braquicéfalos, predispondo esses animais a alterações anatômicas das vias aéreas. Geiger \& Haussman (2016) observaram ainda maior número de suturas e sincondroses em crânios de cães braquicefálicos. As possíveis causas que influenciam o desenvolvimento da síndrome, por dificultar o fluxo normal do ar nas vias aéreas, são: ossos do crânio extremamente curtos, narinas alteradas, dorso rotação dos dentes, meato nasal ventral, conchas nasais e ossos etmoidais comprimidos assumindo uma posição vertical (Oechtering, Kiefer \& Noeller, 2008). Acredita-se que as alterações na conformação craniana dos braquicéfalos levem ao estreitamento do espaço intranasal, diminuição da nasofaringe e espaço retrofaríngeo, hiperplasia de mucosa e colapso de vias aéreas anteriores, causando intensa obstrução e levando ao desenvolvimento da síndrome respiratória. (Dupré \& Heidenrich, 2016).

\section{Alterações anatômicas}

Atualmente a síndrome respiratória obstrutiva dos cães braquicefálicos é amplamente encontrada na rotina de clínica médica e cirúrgica, devido à grande popularidade dessas raças (Meola, 2013). As alterações anatômicas primárias, como estenose de narina, prolongamento do palato mole e hipoplasia de traqueia, levam ao estreitamento das vias aéreas. Tais defeitos podem gerar alterações secundárias importantes como eversão de sáculos laríngeos, edema e inflamação de nasofaringe, paralisia e colapso de laringe (Meola, 2013; Dupré \& Heidenrich 2016). Em função da conformação craniana das raças braquicefálicas, outras alterações secundárias que atuam diretamente na fisiopatologia da síndrome obstrutiva, como colapso de brônquio principal, cistos epiglóticos, granulomas de laringe, hiperplasia de amígdalas e macroglossia, são frequentemente encontradas nesses animais (Emmerson, 2014). 


\section{Estenose de narinas e cornetos aberrantes}

Em cães, as narinas e a cavidade nasal apresentam funções extremamente importantes na fisiologia respiratória e no controle da temperatura (Coppola, Craven, Seeger \& Weiler, 2014). Animais portadores de estenose de narinas apresentam significativa redução no espaço aéreo, dificultando a chegada do ar até os pulmões (Torrez \& Hunt, 2006; Oechtering, 2010).

Sabe-se que quanto mais anterior a resistência à entrada do ar, maior o esforço inspiratório (Koch et al., 2003; Meola, 2013). Na Figura 1, pode-se notar a estenose bilateral de narina, apontada pelas setas vermelhas, que por ser a alteração anatômica mais anterior, é um importante ponto de obstrução das vias aéreas, aumentando fortemente a sobrecarga inspiratória (Koch et al., 2003).

Figura 1 - Estenose bilateral de narinas em cão da raça Pug de cinco anos de idade.

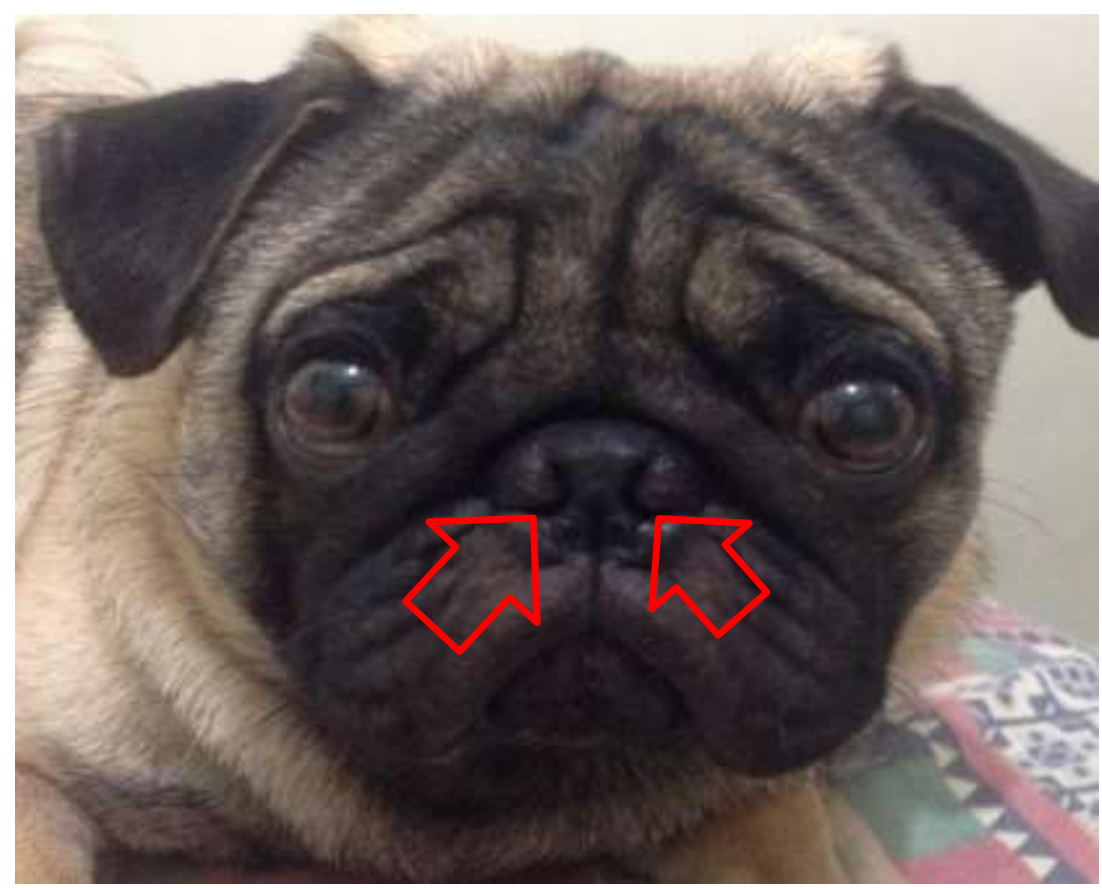

Fonte: Projeto Narizinho, Hospital Universitário de Medicina Veterinária Professor Firmino Mársico Filho - Universidade Federal Fluminense (HUVET- UFF) (2019).

A estenose de narinas é um dos principais elementos obstrutivos da SB e está presente em 50 a $100 \%$ dos casos diagnosticados, exercendo impacto direto na gravidade dos sinais clínicos (Liu, Sargan, Adams \& Ladlow, 2015). Nessa alteração, durante o momento inspiratório, as cartilagens nasais dorsolaterais se deslocam medialmente, diminuindo drasticamente o lúmen do orifício (Poncet, Dupré, Freiche \& Bouvy, 2006; Haimel \& Dupré, 2015; Dupré \& Heidenreich, 2016). Medidas obtidas a partir de imagens de tomografia computadorizada ratificaram achados anteriores e constataram maior resistência ao fluxo do ar em direção aos pulmões na cavidade nasal, quando comparado à região de nasofaringe e palato mole, demonstrando assim a extrema importância deste constituinte na fisiopatologia da doença (Hostnik, Scansen, Zielinski \& Ghadiali, 2017). A obstrução pode se tornar ainda mais pronunciada em função da diminuição do vestíbulo, provocada pelo aumento da porção mais interna da asa da narina (Torrez \& Hunt, 2006; Oechtering, 2010).

Cães braquicefálicos com obstrução grave de cavidade nasal podem apresentar uma resistência de $80 \%$ à passagem de ar (Hoffman, 2007). Porém, além da acentuada obstrução causada pela estenose das narinas, acredita-se que, nos braquicéfalos, o fluxo aéreo intranasal também seja reduzido pela presença dos cornetos (conchas) nasais aberrantes e pelos espaços intranasais reduzidos (Oechtering, Pohl, Schlueter \& Schuenemann, 2016), além do maior contato intramucosas (Auger, Alexander, Beauchamp \& Dunn, 2016; Oechtering, Pohl, Schlueter \& Schuenemann, 2016). 
Na Figura 2, nota-se estrutura de contornos irregulares e superfície lisa, e corneto nasal aberrante posterior (seta), causando obstrução de coana esquerda.

Figura 2 - Imagem de rinoscopia retrógrada da região de nasofaringe posterior de cão da raça Buldogue francês de dois anos de idade. Corneto aberrante caudal(seta).

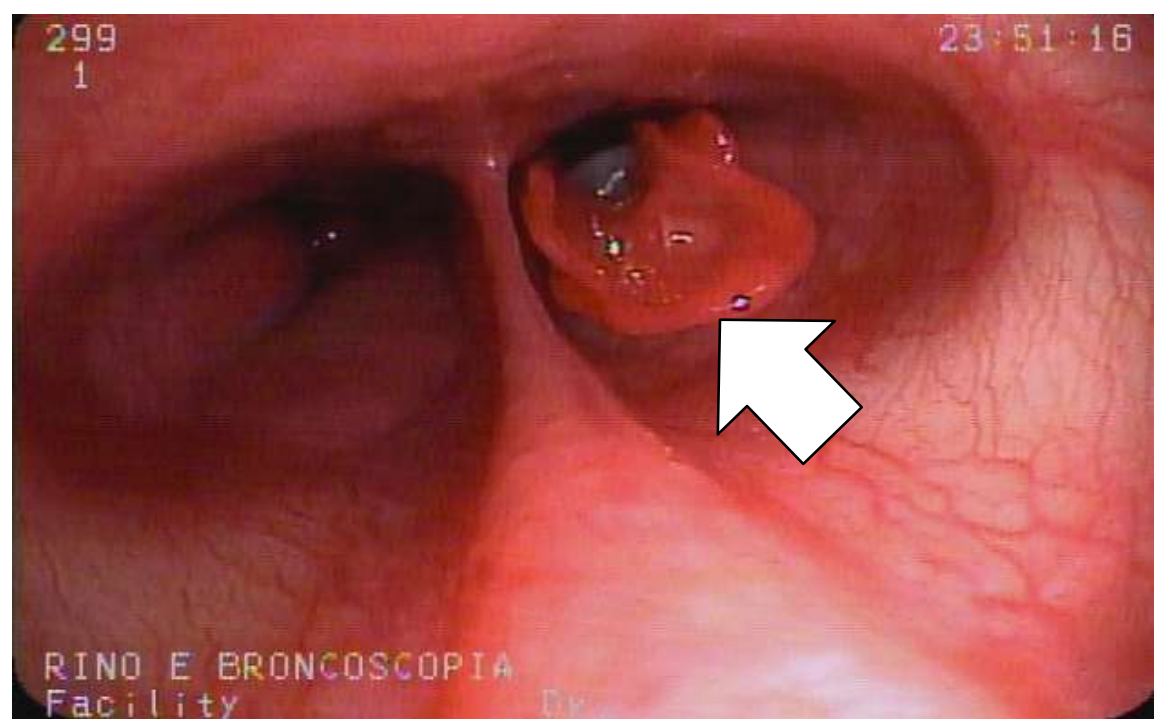

Fonte: Dra Flavia Toledo. Rio de Janeiro (2020).

Os cornetos são responsáveis pela umidificação e aquecimento do ar para a chegada aos pulmões. Com a diminuição do lúmen intranasal, nos cães braquicefálicos, não há espaço suficiente para o desenvolvimento dos cornetos, o que acarreta um crescimento anormal dessas estruturas e o surgimento dos cornetos aberrantes que aumentam os pontos de contato entre as mucosas nasais. São encontrados dois tipos de crescimento aberrante: os cornetos aberrantes rostrais (RAT), que causam obstrução da passagem nasal, e os cornetos aberrantes caudais, que obstruem a nasofaringe, aumentando o esforço inspiratório (Oechtering, Oechtering \& Nöller, 2007; Vilaplana Grosso, Harr \& Boroffka, 2015).

É grande a prevalência de cornetos aberrantes nos animais com o crânio diminuído. Oechtering, Pohl, Schlueter, Lippert et al. (2016) constataram a presença de cornetos aberrantes rostrais em $90 \%$ dos Pugs, 56,4\% dos Buldogues franceses e 36,4\% dos Buldogues ingleses. Foi revelada ainda a presença de cornetos aberrantes caudais em 66,7\% desses animais, e todos mostraram vários pontos de contato entre as mucosas, aumentando a obstrução intranasal e intracoanas. Os autores justificaram também que, em consequência do nariz mais encurtado, os cães da raça Pug apresentaram mais alterações obstrutivas na cavidade nasal.

\section{Prolongamento e espessamento de palato mole}

O prolongamento do palato mole (Figura 3) é considerado uma alteração primária na SB (Sławuta; Glińska-Suchocka \& Cekiera, 2015). Durante a inspiração, quando o ar é recrutado pela cavidade oral, o palato mole alongado reduz o lúmen da nasofaringe e da orofaringe, aumentando a resistência do fluxo do ar pelas vias aéreas (Pichetto, Arrighi, Roccabianca \& Romussi, 2011). 
Figura 3 - Prolongamento de palato mole em cão da raça Buldogue francês, um ano de idade. Palato mole ultrapassando epiglote (seta).

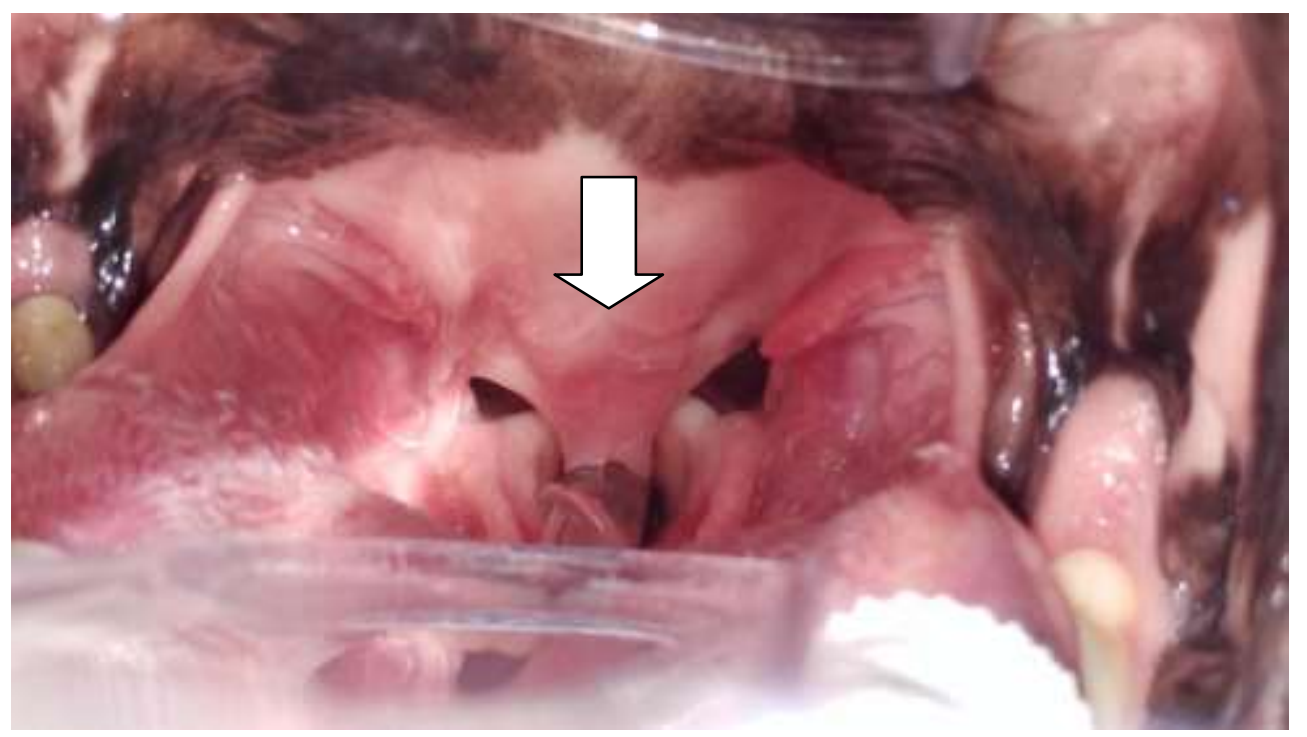

Fonte: Projeto Narizinho, Hospital Universitário de Medicina Veterinária Professor Firmino Mársico Filho - Universidade Federal Fluminense (HUVET- UFF) (2019).

A avaliação histológica do palato mole de cães braquicefálicos evidenciou alterações como hiperplasia do epitélio superficial, grandes ondulações de estrias interfibrilar, edema da lâmina própria, edema de mucosa, hiperplasia de glândulas e músculos e degeneração estrutural de fibras (Pichetto et al., 2011). Ainda quando equiparado histologicamente ao de mesaticefálicos, o palato mole de cães braquicefálicos apresentou aumento acentuado da degeneração muscular aguda e crônica, necrose, atrofia de miofibrila, perda de estrias cruzadas, sarcoplasma fragmentado, aumento de estroma de colágeno e aumento de tecido de glândula salivar (Crosse, Bray, Orbell \& Preston, 2015).

Um estudo recente realizou ressonância magnética do crânio de cães braquicefálicos e não braquicefálicos, comparando seu volume e as imagens tridimensionais, e confirmando que os cães braquicefálicos apresentam o palato com volume e espessura maior que o dos cães mesaticefálicos, o que influencia diretamente a redução do espaço nasofaríngeo, favorecendo a ocorrência de um colapso nasofaríngeo (Kim et al., 2018).

Alterações secundárias do palato mole podem surgir em função do edema e da inflamação causados por microtraumas secundários ao aumento da turbulência na região da orofaringe, pela elevação da pressão negativa e pelo consequente aumento do esforço inspiratório, levando a alterações fibromusculares e aumentando a intensidade dos sinais clínicos (Kim et al., 2018). Esse fato foi comprovado pela avaliação do palato mole de neonatos natimortos, quando se constatou que, diferentemente do palato de animais braquicefálicos adultos, o palato de cães neonatos não mostrava sinais de edema e demais alterações histológicas, reforçando o cunho progressivo da síndrome (Pichetto, Arrighi, Gobbetti \& Romussi, 2015). Existe uma correlação positiva entre a gravidade dos sinais clínicos e o tamanho e espessura do palato, mostrando que animais com sintomatologia mais grave apresentam o palato maior e mais espesso (Heidenreich, Gradner, Kneissl \& Dupré, 2016).

\section{Hipoplasia de traqueia}

A hipoplasia de traqueia é caracterizada por uma redução do diâmetro de todo o lúmen do órgão. Essa alteração é encontrada com frequência em pacientes braquicefálicos, especialmente em cães da raça Buldogue inglês (Koch et al., 2003; Ingman, Naslund \& Hansson, 2014; Kaye, Boroffka, Haagsman \& Ter Haar, 2015). Recentemente, mediante a avaliação de 
exames radiográficos, observou-se que cães com características genéticas braquicefálicas mais expressivas apresentam um percentual maior de ocorrência de hipoplasia de traqueia (Komsta, Osiński, Debiak, Twardowski \& Lisiak, 2019).

A etiologia da hipoplasia de traqueia difere nas principais raças de prevalência. Nos Pugs, essa anomalia ocorre como resultado de traqueomalácia, que ocasiona maior flacidez estrutural e achatamento dos anéis cartilaginosos secundários à degeneração. Já nos Buldogues, a hipoplasia manifesta-se em função da presença de anéis cartilaginosos mais rígidos e de diâmetro excessivamente reduzido. Em muitos casos, pode ser observada a associação da hipoplasia de traqueia com o colapso brônquico (Oechtering, 2010, Yoon et al., 2020).

Apesar de não haver correlação direta com um pior resultado pós-cirúrgico, sabe-se que, quando associada às demais alterações anatômicas da SB, a hipoplasia de traqueia tem impacto direto nas manifestações clínicas e na qualidade de vida dos animais (Koch et al., 2003; Ingman et al. 2014; Kaye et al., 2015).

\section{Eversão de sáculos laríngeos}

Em consequência do aumento da pressão negativa nas regiões da faringe, laringe e tórax, provocada pelas obstruções primárias, pode ocorrer a eversão dos sáculos laríngeos, observadas como massas teciduais rostrais às cordas vocais (Koch et al., 2003; Caccamo, Buracco, La Rosa, Cantatore \& Romussi, 2014). Trata-se do primeiro dos três estágios do colapso de laringe (Packer \& Tivers, 2015). O segundo estágio é caracterizado pelo desvio medial dos processos cuneiformes das cartilagens aritenóides, e o terceiro, pelo colapso dos processos corniculados, com perda do arco dorsal da rima glótica (Koch et al., 2003, Bowlt \& Moore, 2009; Packer \& Tivers, 2015).

Figura 4 - Eversão de sáculos laríngeos.

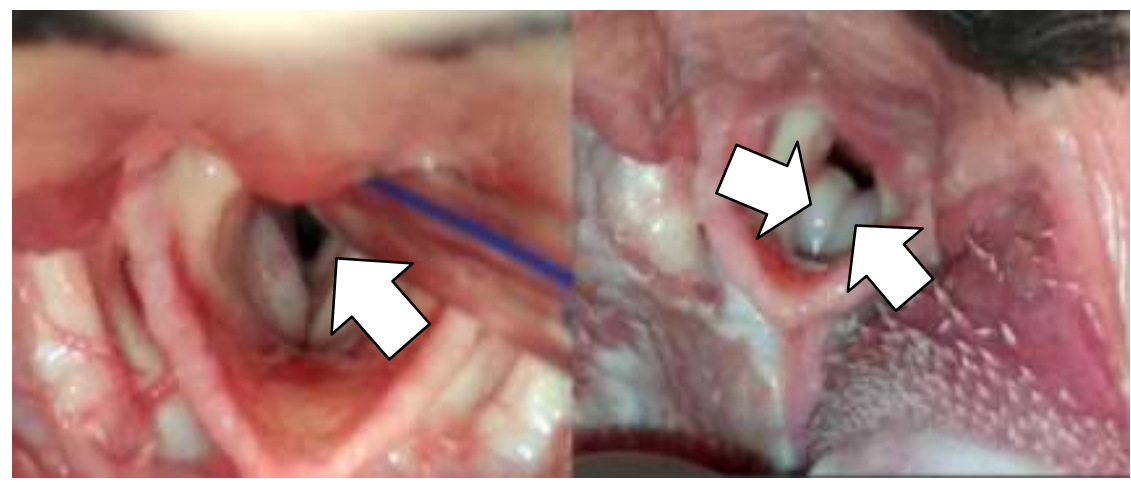

Fonte: Projeto Narizinho, Hospital Universitário de Medicina Veterinária Professor Firmino Mársico Filho - Universidade Federal Fluminense (HUVET- UFF) (2019).

A eversão é um achado comum nas raças braquicefálicas, reconhecida em 40 a $60 \%$ dos exemplares (Gianella et al., 2019). Em estudo recente da nasofaringe de cães braquicefálicos, revelou que $85 \%$ dos cães analisados apresentavam colapso laríngeo no grau I, e 33\% no grau 3 (Kim et al., 2018). Essas alterações impactam diretamente o prognóstico e o tratamento do paciente (Cantatore et al., 2012), pois dificultam significativamente a passagem do ar pela laringe, podendo intensificar a apresentação clínica da doença (Dupré \& Heidenreich, 2016).

A progressão da doença indicada pela eversão dos sáculos laríngeos é preditiva de um prognóstico reservado para o caso, podendo ser necessária a ressecção dessas estruturas mediante procedimento cirúrgico corretivo (Cantatore et al., 2012).

\section{Sinais clínicos}

Os cães braquicefálicos acometidos pela síndrome apresentam importante perda no que se refere ao bem-estar e à qualidade de vida, com elevado risco de óbito (Emmerson, 2014). A sintomatologia se desenvolve em conformidade com a 
fisiopatologia da doença, e sua gravidade varia de acordo com o nível de obstrução das vias aéreas anteriores (Oechtering, 2010; Packer \& Tivers, 2015; Ladlow, Liu, Kalmar \& Sargan, 2018).

Os sinais clínicos podem se iniciar a partir dos primeiros meses de idade, e perdurar ao longo da vida, alguns deles persistindo mesmo após o tratamento clínico e cirúrgico (Dupré \& Heidenreich, 2016). Alterações respiratórias, gastrintestinais e cardiovasculares são frequentemente encontradas (Emmerson, 2014; Packer \& Tivers, 2015; Liu, Troconis et al., 2017). Quando expostos a exercícios físicos extenuantes e altas temperaturas, acontece a exacerbação desses sinais, aumentando o risco para esses animais (Trappler \& Moore, 2011; Dupré \& Heidenreich, 2016).

\section{Alterações respiratórias}

Certamente as manifestações clínicas respiratórias são as mais observadas em cães braquicefálicos afetados pela síndrome (O’Neill et al., 2015). Dispneia, cianose, tosse, espirro, espirro reverso, ronco, intolerância ao exercício, ruídos respiratórios alterados, doenças pulmonares crônicas e apneia são sinais comumente relatados (Meola, 2013; Emmerson, 2014; Mendes Junior, Silva, Soares \& Almosny, 2017; Liu, Oechtering et al., 2017).

Em consequência do intenso processo obstrutivo das vias aéreas anteriores, principalmente da estenose das narinas, que aumenta grandemente a resistência à entrada do ar, a dispneia inspiratória padrão obstrutivo é um sinal clínico de destaque normalmente encontrado nos animais (Roedler, Pohl \& Oechtering, 2013). O aumento da gravidade dos quadros dispneicos pode ser percebido em casos de progressão da doença e associado a alterações de laringe, traqueia e intermação (Johnson, 2016; Tappin, 2016; Bruchim, Horowitz \& Aroch, 2017).

Acredita-se que a alta prevalência do ronco em raças braquicefálicas se deve à acentuada diminuição da região faríngea associada ao palato mole alongado, que ocasionam aumento do esforço inspiratório e da pressão negativa, acarretando grande turbulência e vibração nessa estrutura, o que produz reverberação e, consequentemente, o ronco (Schuenemann \& Oechtering, 2014; Oechtering, Pohl, Schlueter, Lippert et al.,2016; Seneviratne, Kaye \& Ter Haar, 2020). Outras alterações comumente identificadas nos braquicéfalos como tonsilas hipertrofiadas e macroglossia, também influenciam diretamente a ocorrência do ronco nesses animais, pois diminuem ainda mais o lúmen da orofaringe (Schuenemann \& Oechtering, 2014; Oechtering, Pohl, Schlueter, Lippert et al.,2016, confirmando uma relação entre o grau de estenose da nasofaringe e a intensidade do ronco em cães braquicefálicos (Seneviratne et al., 2020).

No estudo de Mendes Junior et al., (2017), todos os tutores dos animais avaliados relataram que esses animais apresentavam ronco dormindo (100\%) e ronco quando acordados (80,7\%). Anteriormente, Torrez e Hunt (2006) também apuraram que uma alta percentagem $(71 \%)$ de cães braquicefálicos apresentava ronco dormindo.

Semelhante ao que acontece em pacientes humanos portadores de obstruções em vias aéreas anteriores, os cães braquicefálicos são extremamente propensos ao desenvolvimento de distúrbios do sono (Planellas et al., 2012). Os responsáveis descrevem episódios de apneia em seus animais, principalmente no período noturno (Slawuta, Nicpon \& Domanska, 2011; Planellas, et al., 2012; Mendes Junior et al., 2017). Esse fato se deve provavelmente ao maior relaxamento muscular das estruturas no período noturno, que exacerba a obstrução das vias respiratórias (Planellas et al., 2015).

Foi confirmado, por meio de tromboelastografia e determinação da contagem de hematócritos e plaquetas em Buldogues ingleses saudáveis, a ocorrência de hipercoagulabilidade, similarmente ao que acontece em seres humanos portadores da apneia obstrutiva do sono (Hoareau \& Mellema, 2015).

A obstrução das vias aéreas causa aumento da pressão negativa intratorácica, o que, por sua vez, promove o aumento no retorno venoso para o lado direito do coração, elevando a pressão hidrostática capilar pulmonar, seguida da transudação de líquido para os alvéolos, e causando edema pulmonar agudo não cardiogênico $\mathrm{O}$ edema pulmonar pode se tornar uma emergência, e é considerado uma das causas de morte súbita em braquicéfalos. Ocorre principalmente nos momentos de 
angústia respiratória gerada pela obstrução das vias aéreas anteriores, secundária a exercícios físicos extenuados ou em temperaturas elevadas (Shales, 2014).

\section{Distúrbios gastrintestinais}

A etiologia de uma gama de alterações de trato gastrintestinal em cães braquicefálicos está diretamente associada à obstrução das vias aéreas anteriores. Normalmente são constatados sinais como disfagia, engasgo, regurgitação, flatulência, vômito, esofagite e gastrite (Roedler et al., 2013; Dupré \& Heidenreich, 2016; Eivers, Rueda, Liuti \& Schmitz, 2019). O grau de obstrução e a obesidade são fatores de risco associados à ocorrência e à gravidade dos sinais gastrintestinais (Poncet et al., 2005; Poncet et al., 2006; Liu et al., 2015).

No estudo realizado por Poncet et al. (2005), 98\% dos cães braquicefálicos apresentavam algum tipo de alteração gastrintestinal. Desses, 37\% sofriam de esofagite, 89\% de inflamação gástrica difusa, e 53\% de inflamação difusa de duodeno. Os resultados da avaliação histológica identificaram ainda que 98\% dos cães apresentavam lesões compatíveis com gastrite folicular difusa crônica e 97,7 \% com duodenite linfocítica.

Em muitos casos, quando o grau de obstrução de vias aéreas anteriores é grave e o animal necessita realizar respiração oral, é comum a ocorrência de distensão abdominal e de flatulência, em consequência da aerofagia (Roedler et al., 2013). O refluxo gastrintestinal que, juntamente com os quadros de regurgitação e vômito, pode ocasionar laringite, faringite e esofagite, acontece pelo aumento da pressão negativa torácica secundária ao aumento do esforço inspiratório necessário para vencer a obstrução das vias aéreas (Roedler et al., 2013; Shaver et al., 2017).

Distúrbios de motilidade esofágica foram associados à síndrome braquicefálica e um total de 30 cães de variadas raças braquicefálicas ou não, 26 apresentaram disfunções de motilidade esofágica. Destes, 77\% eram cães de raças braquicefálicas, dos quais oito apresentaram hérnia hiatal (Eivers et al., 2019). Outro estudo recente detectou sintomatologia relacionada ao sistema digestório em 93\% dos Buldogues franceses, 58\% dos Buldogues ingleses e 16\% dos cães da raça Pug (Fenner, Quinn \& Demetriou, 2019).

\section{Intermação}

A propensão dos braquicéfalos a desenvolver hipertermia se deve à falha primária da termorregulação periférica, que acontece de maneira secundária aos defeitos anatômicos, e não por mecanismos centrais (Oechtering, 2010). Percebe-se nesses animais um lapso nos mecanismos de inspiração e expiração, levando a uma baixa troca de calor e resfriamento ineficaz. A SB é apontada como fator de risco para o desdobramento da intermação, podendo levar os animais à morte (Oechtering et al., 2007; Bruchim et al., 2016; Bruchim et al., 2017).

A intermação é uma desordem aguda, influenciada por fatores externos, como estresse, temperatura ambiente de moderada a alta e exercícios físicos fatigantes. Nesses episódios, constata-se nos animais temperatura corporal acima de $41^{\circ} \mathrm{C}$, em virtude da falha na dissipação do calor, que leva a um desequilíbrio na termorregulação, com disfunção do sistema nervoso central associada à ocorrência da síndrome da resposta inflamatória sistêmica (Bruchim et al., 2017).

\section{Disfunções cardiovasculares}

Acredita-se que alterações cardiovasculares possam surgir em cães braquicefálicos de modo secundário à doença obstrutiva. Desse modo, sinais clínicos, como síncope, arritmias, mucosas cianóticas, dispneia e intolerância ao exercício podem ser encontrados (Camacho, 2006).

Humanos diagnosticados com apneia obstrutiva do sono são susceptíveis a episódios hipertensivos, em virtude de maior atividade simpática, maior dano endotelial, diminuição da produção de óxido nítrico e aumento da pressão intratorácica 
negativa, provocando a elevação da pressão arterial (Gonzaga, Bertolami, Bertolami, Amodeo \& Calhoun, 2015). Considera-se que, em função da síndrome respiratória, esses episódios possam ocorrer nos braquicéfalos (Hoffman, 2007). Com o objetivo de avaliar a predisposição ao desenvolvimento de hipertensão arterial sistêmica, cães braquicefálicos foram submetidos a aferições da pressão arterial sistólica, média e diastólica, posteriormente comparadas com os valores de não braquicefálicos. Essas aferições mostraram que os cães braquicefálicos apresentam valores de pressão arterial sistólica dentro da normalidade, porém superiores quando comparados aos animais de outras raças (Hoareau, Jourdan, Mellema \& Verwaerde, 2012).

O aumento no tônus vagal, em função do colapso das vias aéreas, pode induzir em animais braquicefálicos a episódios de parada ou bradicardia sinusal (Shales, 2014). O aumento da ativação parassimpática sobre o batmotropismo cardíaco em cães braquicefálicos causa importantes distúrbios de ritmo cardíaco, aumentando a variabilidade da frequência cardíaca pelo estímulo da atividade parassimpática na regulação autonômica do coração (Santos Filho et al., 2020).

A hipertensão pulmonar (HP), definida como um aumento anormal e persistente da pressão arterial pulmonar (Rich, Braunwald \& Grossman, 1997), se desenvolve principalmente de forma secundária a doenças cardiopulmonares, como tromboembolia, cardiopatia congênita, vasculite pulmonar, dirofilariose, neoplasias, além de alterações respiratórias, como a obstrução de vias aéreas (Winter et al., 2017). Em função de sua anatomia característica, presume-se que os cães braquicefálicos apresentem grande propensão ao desenvolvimento de HP, uma importante repercussão cardiovascular que pode ocorrer secundária à síndrome do braquicéfalo em cães portadores da doença (Camacho, 2006).

A hipertensão arterial pulmonar vasoclusiva, que pode se desenvolver em braquicefálicos (Camacho, 2006), é reconhecida em quadros obstrutivos que cursam com vasoconstrição das artérias pulmonares e causam o aumento da resistência vascular periférica, podendo levar à sobrecarga do ventrículo direito (Kellihan \& Stepien, 2010).

A hipertensão pulmonar cursa com vasoconstrição pulmonar e remodelamento vascular. Em situações em que o aumento da pressão pulmonar altera a estrutura e a função do ventrículo direito, tem-se um quadro denominado Cor Pulmonale, que pode causar insuficiência ventricular direita e até mesmo, o óbito do paciente (Lapa et al., 2006).

Em conformidade com o processo fisiopatológico obstrutivo e o caráter progressivo e crônico da doença, acredita-se que os cães braquicefálicos sejam altamente suscetíveis à ocorrência de hipertensão pulmonar e, portanto, ao desenvolvimento de Cor Pulmonale (Hoareau et al., 2012), em função da hipóxia crônica alveolar e a consequente vasoconstrição arterial précapilar, que leva ao aumento da pressão da artéria pulmonar e à possível hipertrofia ventricular direita (Allen \& Mackin, 2001, Fasanella, Shivley, Wardla \& Givaruangsawat, 2010).

De extrema relevância entre as doenças cardiovasculares a que os braquicéfalos são predispostos, à hipertensão pulmonar ganha destaque pelo prognóstico reservado, sendo importante o diagnóstico precoce, com o objetivo de melhorar a qualidade de vida e aumentar a sobrevida desses animais (Hoareau et al., 2012).

\section{Considerações Finais}

Os dados levantados salientam a importância do conhecimento acerca das alterações anatômicas e manifestações clínicas da síndrome braquicefálica pelo médico veterinário para o diagnóstico precoce e indicação do tratamento adequado, proporcionando assim melhor qualidade de vida e bem estar ao animal, sendo assim o presente trabalho permitiu comparar os resultados dos diferentes estudiosos do assunto, permitindo maior entendimento, para melhor identificação do paciente acometido pela síndrome braquicefálica.

Assim sendo sugere-se a produção de trabalhos que relacionem os aspectos anatômicos com a resposta terapêutica do tratamento para o paciente portador da SB. 


\section{Referências}

Allen, D. G. \& Mackin. A. (2001). Cor pulmonale. In: Tilley, L. P. \& Goodwin, J. K (Eds.). Manual of Canine and Feline Cardiology. 3rd ed., Philadelphia, Saunders, 197-214.

Auger, M., Alexander, K., Beauchamp, G., \& Dunn, M. (2016). Use of CT to evaluate and compare intranasal features in brachycephalic and normocephalic dogs. The Journal of small animal practice, 57(10), 529-536. https://doi.org/10.1111/jsap.12541

Bowlt, K., \& Moore, A. H. (2009). Surgery of the upper respiratory tract Part 2: Brachycephalic obstructive airway syndrome (BOAS). UK Vet Companion Animal, 14(8), 19-26.

Bruchim, Y., Segev, G., Kelmer, E., Codner, C., Marisat, A., \& Horowitz, M. (2016). Hospitalized dogs recovery from naturally occurring heatstroke; does serum heat shock protein 72 can provide prognostic biomarker? Cell stress \& chaperones, 21(1), 123-130. https://doi.org/10.1007/s12192-015-0645-5

Bruchim, Y., Horowitz, M., \& Aroch, I. (2017). Pathophysiology of heatstroke in dogs-revisited. Temperature, 4(4), 356-370. https://doi.org/10.1080/23328940.2017.1367457

Caccamo, R., Buracco, P., La Rosa, G., Cantatore, M., \& Romussi, S. (2014). Glottic and skull indices in canine brachycephalic airway obstructive syndrome. BMC veterinary research, 10(1), 1-7. https://doi.org/10.1186/1746-6148-10-12

Camacho, A. A. (2006). Brachycephalic airway syndrome. ANAIS 6th Paulista Congress of Small Animal Veterinary Clinics.

Cantatore, M., Gobbetti, M., Romussi, S., Brambilla, G., Stefanello, D., Giudice, C., \& Grieco, V. (2012). Medium term endoscopic assessment of the surgical outcome following laryngeal saccule resection in brachycephalic dogs. Veterinary Record, 170(20), 518-518. https://doi.org/10.1136/vr.100289

Coppola, D. M., Craven, B. A., Seeger, J., \& Weiler, E. (2014). The effects of naris occlusion on mouse nasal turbinate development. Journal of Experimental Biology, 217(12), 2044-2052.

Crosse, K. R., Bray, J. P., Orbell, G. M. B., \& Preston, C. A. (2015). Histological evaluation of the soft palate in dogs affected by brachycephalic obstructive airway syndrome. New Zealand Veterinary Journal, 63(6), 319-325. https://doi.org/10.1080/00480169.2015.1061464

Dupré, G., \& Heidenreich, D. (2016). Brachycephalic Syndrome. The Veterinary clinics of North America. Small animal practice, 46(4), 691-707. https://doi.org/10.1016/j.cvsm.2016.02.002

Eivers, C., Chicon Rueda, R., Liuti, T., \& Salavati Schmitz, S. (2019). Retrospective analysis of esophageal imaging features in brachycephalic versus non-brachycephalic dogs based on videofluoroscopic swallowing studies. Journal of veterinary internal medicine, $33(4), 1740-1746$.

Emmerson T. (2014). Brachycephalic obstructive airway syndrome: a growing problem. The Journal of small animal practice, 55(11), 543-544. https://doi.org/10.1111/jsap.12286

Fasanella, F. J., Shivley, J. M., Wardlaw, J. L., \& Givaruangsawat, S. (2010). Brachycephalic airway obstructive syndrome in dogs: 90 cases (1991-2008). Journal of the American Veterinary Medical Association, 237(9), 1048-1051. https://doi.org/10.2460/javma.237.9.1048

Fenner, J. V., Quinn, R. J., \& Demetriou, J. L. (2020). Postoperative regurgitation in dogs after upper airway surgery to treat brachycephalic obstructive airway syndrome: 258 cases (2013-2017). Veterinary Surgery, 49(1), 53-60. https://doi.org/10.1111/vsu.13297

Geiger, M., \& Haussman, S. (2016). Cranial suture closure in domestic dog breeds and its relationships to skull morphology. The Anatomical Record, 299(4), 412-420.https://doi.org/10.1002/ar.23313

Gianella, P., Caccamo, R., Bellino, C., Bottero, E., Fietta, F., Roncone, S., Ostanello, F., Pietra, M. \& Buracco, P. (2019). Evaluation of metabolic profile and C-reactive protein concentrations in brachycephalic dogs with upper airway obstructive syndrome. Journal of veterinary internal medicine, 33(5), $2183-2192$. https://doi.org/10.1111/jvim.15575

Gonzaga, C., Bertolami, A., Bertolami, M., Amodeo, C., \& Calhoun, D. (2015). Obstructive sleep apnea, hypertension and cardiovascular diseases. Journal of human hypertension, 29(12), 705-712.

Haimel, G., \& Dupré, G. (2015). Brachycephalic airway syndrome: a comparative study between pugs and French bulldogs. Journal of Small Animal Practice, 56(12), 714-719. https://doi.org/10.1111/jsap. 12408

Heidenreich, D., Gradner, G., Kneissl, S., \& Dupré, G. (2016). Nasopharyngeal dimensions from computed tomography of pugs and French bulldogs with brachycephalic airway syndrome. Veterinary Surgery, 45(1), 83-90. https://doi.org/10.1111/vsu.12418

Hoareau, G. L., Jourdan, G., Mellema, M., \& Verwaerde, P. (2012). Evaluation of arterial blood gases and arterial blood pressures in brachycephalic dogs. Journal of Veterinary Internal Medicine, 26(4), 897-904.

Hoareau, G., \& Mellema, M. (2015). Pro-coagulant thromboelastographic features in the bulldog. Journal of Small Animal Practice, $56(2), 103-107$.

Hoffman, A. M. (2007). Airway physiology and clinical function testing. Veterinary Clinics of North America: Small Animal Practice, 37(5), 829-843.

Hostnik, E. T., Scansen, B. A., Zielinski, R., \& Ghadiali, S. N. (2017). Quantification of nasal airflow resistance in English bulldogs using computed tomography and computational fluid dynamics. Veterinary Radiology \& Ultrasound, 58(5), 542-551.

Ingman, J., Näslund, V., \& Hansson, K. (2014). Comparison between tracheal ratio methods used by three observers at three occasions in English Bulldogs. Acta Veterinaria Scandinavica, 56(1), 1-7. https://doi.org/10.1186/s13028-014-0079-6 
Johnson, L. R. (2016). Laryngeal structure and function in dogs with cough. Journal of the American Veterinary Medical Association, 249(2), 195-201. https://doi.org/10.2460/javma.249.2.195

Kaye, B. M., Boroffka, S. A., Haagsman, A. N., \& Haar, G. T. (2015). Computed tomographic, radiographic, and endoscopic tracheal dimensions in English bulldogs with Grade 1 clinical signs of brachycephalic airway syndrome. Veterinary Radiology \& Ultrasound, 56(6), 609-616, https://doi.org/10.1111/vru.12277

Kellihan, H. B., \& Stepien, R. L. (2010). Pulmonary hypertension in dogs: diagnosis and therapy. Veterinary Clinics: Small Animal Practice, 40 (4), $623-641$.

Kim, Y. J., Lee, N., Yu, J., Lee, H., An, G., Bang, S., Chang, J. \& Chang, D. (2018). Three-dimensional volumetric magnetic resonance imaging (MRI) analysis of the soft palate and nasopharynx in brachycephalic and non-brachycephalic dog breeds. Journal of Veterinary Medical Science, 17-0711. https://doi.org/10.1292/jvms.17-0711

Koch, D. A., Arnold, S., Hubler, M., \& Montavon, P. M. (2003). Brachycephalic syndrome in dogs. Compendium On Continuing Education For The Practising Veterinarian - North American Edition, 25(1), 48-55.

Komsta, R., Osiński, Z., Dębiak, P., Twardowski, P., \& Lisiak, B. (2019). Prevalence of pectus excavatum (PE), pectus carinatum (PC), tracheal hypoplasia, thoracic spine deformities and lateral heart displacement in thoracic radiographs of screw-tailed brachycephalic dogs. Plos one, 14(10). https://doi.org/10.1371/journal.pone.0223642

Ladlow, J., Liu, N. C., Kalmar, L., \& Sargan, D. (2018). Brachycephalic obstructive airway syndrome. Veterinary Record, 182(13), 375-378, https://doi.org/10.1136/vr.k1403

Lapa, M. S., Ferreira, E. V. M., Jardim, C., Martins, B. D. C. D. S., Arakaki, J. S. O., \& Souza, R. (2006). Características clínicas dos pacientes com hipertensão pulmonar em dois centros de referência em São Paulo. Revista da Associação Médica Brasileira, 52, 139-143. https://doi.org/10.1590/S010442302006000300012

Liu, N. C., Sargan, D. R., Adams, V. J., \& Ladlow, J. F. (2015). Characterisation of brachycephalic obstructive airway syndrome in French bulldogs using whole-body barometric plethysmography. PLoS One, 10(6), e0130741. https://doi.org/10.1371/journal.pone.0130741

Liu, N. C., Troconis, E. L., Kalmar, L., Price, D. J., Wright, H. E., Adams, V. J., Sargan, D. R., \& Ladlow, J. F. (2017). Conformational risk factors of brachycephalic obstructive airway syndrome (BOAS) in pugs, French bulldogs, and bulldogs. PloS one, 12(8), e0181928. https://doi.org/10.1371/journal.pone.0181928

Liu, N. C., Oechtering, G. U., Adams, V. J., Kalmar, L., Sargan, D. R., \& Ladlow, J. F. (2017). Outcomes and prognostic factors of surgical treatments for brachycephalic obstructive airway syndrome in 3 breeds. Veterinary Surgery, 46(2), 271-280.https://doi.org/10.1111/vsu.12608

Mendes Junior, A., Silva, G. S., Soares, A. M., \& Almosny, N. R. (2017). Percepção de tutores quanto aos sinais clínicos em cães braquicefálicos portadores de estenose de narina. Enciclopédia Biosfera, 14(26).

Meola, S. D. (2013). Brachycephalic airway syndrome. Topics in companion animal medicine, 28(3), 91-96.

O’Neill, D. G., Jackson, C., Guy, J. H., Church, D. B., McGreevy, P. D., Thomson, P. C., \& Brodbelt, D. C. (2015). Epidemiological associations between brachycephaly and upper respiratory tract disorders in dogs attending veterinary practices in England. Canine genetics and epidemiology, 2(1), 1-10. https://doi.org/10.1186/s40575-015-0023-8

Oechtering, G. (2010). Síndrome braquicefálica: novas informações sobre uma antiga doença congênita. Veterinary Focus, 20(2), 10-18.

Oechtering, G. U., Pohl, S., Schlueter, C., \& Schuenemann, R. (2016). A Novel Approach to Brachycephalic Syndrome. 2. Laser-Assisted Turbinectomy (LATE). Veterinary surgery: VS, 45(2), 173-181. https://doi.org/10.1111/vsu.12447

Oechtering, G. U., Pohl, S., Schlueter, C., Lippert, J. P., Alef, M., Kiefer, I., \& Schuenemann, R. (2016). A novel approach to brachycephalic syndrome. 1. Evaluation of anatomical intranasal airway obstruction. Veterinary Surgery, 45(2), 165-172.

Oechtering, G. U., Kiefer, I., \& Noeller, C. (2008). Brachycephalic airway syndrome, part 1: A New Understanding - It is an intranasal problem. The North American Veterinary Conference, 1450-1451.

Oechtering, T. H., Oechtering, G. U., \& Nöller, C. (2007). Structural characteristics of the nose in brachycephalic dog breeds analysed by computed tomography. Tierärztliche Praxis Ausgabe K: Kleintiere/Heimtiere, 35(03), 177-187. https://doi.org/10.1055/s-0038-1622615

Packer, R. M., \& Tivers, M. S. (2015). Strategies for the management and prevention of conformation-related respiratory disorders in brachycephalic dogs. Veterinary medicine (Auckland, N.Z.), 6, 219-232. https://doi.org/10.2147/VMRR.S60475

Packer, R. M., Hendricks, A., Tivers, M. S., \& Burn, C. C. (2015). Impact of Facial Conformation on Canine Health: Brachycephalic Obstructive Airway Syndrome. PloS one, 10(10), e0137496. https://doi.org/10.1371/journal.pone.0137496

Pichetto, M., Arrighi, S., Gobbetti, M., \& Romussi, S. (2015). The anatomy of the dog soft palate. III. Histological evaluation of the caudal soft palate in brachycephalic neonates. The Anatomical Record, 298(3), 618-623. https://doi.org/10.1002/ar.23054

Pichetto, M., Arrighi, S., Roccabianca, P., \& Romussi, S. (2011). The anatomy of the dog soft palate. II. Histological evaluation of the caudal soft palate in brachycephalic breeds with grade I brachycephalic airway obstructive syndrome. The Anatomical Record: Advances in Integrative Anatomy and Evolutionary Biology, 294(7), 1267-1272. https://doi.org/10.1002/ar.21417

Planellas, M., Cuenca, R., Tabar, M. D., Bertolani, C., Poncet, C., Closa, J. M., Lorente, J., Cerón, J. J., \& Pastor, J. (2012). Evaluation of C-reactive protein, haptoglobin and cardiac troponin 1 levels in brachycephalic dogs with upper airway obstructive syndrome. BMC veterinary research, $8,152$. https://doi.org/10.1186/1746-6148-8-152 
Planellas, M., Cuenca, R., Tabar, M. D., Bertolani, C., Poncet, C., Closa, J. M., Lorente, J., Cerón, J. J., \& Pastor, J. (2015). Clinical assessment and C-reactive protein (CRP), haptoglobin (Hp), and cardiac troponin I (cTnI) values of brachycephalic dogs with upper airway obstruction before and after surgery. Canadian Journal of Veterinary Research, 79(1), 58-63.

Poncet, C. M., Dupre, G. P., Freiche, V. G., Estrada, M. M., Poubanne, Y. A., \& Bouvy, B. M. (2005). Prevalence of gastrointestinal tract lesions in 73 brachycephalic dogs with upper respiratory syndrome. The Journal of small animal practice, 46(6), 273-279. https://doi.org/10.1111/j.17485827.2005.tb00320.x

Poncet, C. M., Dupre, G. P., Freiche, V. G., \& Bouvy, B. M. (2006). Long-term results of upper respiratory syndrome surgery and gastrointestinal tract medical treatment in 51 brachycephalic dogs. The Journal of small animal practice, 47(3), 137-142. https://doi.org/10.1111/j.1748-5827.2006.00057.x

Rich S., Braunwald E., \& Grossman W. (1997). Pulmonary hypertension. In: Braunwald E. (editors). Heart disease. 5th ed. Philadelphia: WB Saunders, 780806.

Roedler, F. S., Pohl, S., \& Oechtering, G. U. (2013). How does severe brachycephaly affect dog's lives? Results of a structured preoperative owner questionnaire. Veterinary journal (London, England : 1997), 198(3), 606-610. https://doi.org/10.1016/j.tvj1.2013.09.009

Santos Filho, M. dos; Hainfellner, D. C., Lemos, N. M. de O., Macambira, K. D. da S. M. B., Do Carmo, J. S., Alberigi, B. R. S. A. da S., Aben Athar, C. do V., Mendes Junior, A. F., da Veiga, C. C. P., Soares, A. M. B., Botteon, P. de T. L., Fernandes, J. I., \& Paiva, J. P. (2021). Study of the heart rate variability in $\operatorname{dogs}$ with brachycephalic syndrome submitted to rhinoplasty surgery. Brazilian Journal of Veterinary Medicine, 42(1), e104919. https://doi.org/10.29374/10.29374/2527-2179.bjvm104920

Schmidt, M. J., Volk, H., Klingler, M., Failing, K., Kramer, M., \& Ondreka, N. (2013). Comparison of closure times for cranial base synchondroses in mesaticephalic, brachycephalic, and cavalier king charles spaniel dogs. Veterinary Radiology \& Ultrasound, 54(5), 497-503.

Schoenebeck, J. J., \& Ostrander, E. A. (2013). The genetics of canine skull shape variation. Genetics, 193(2), 317-325. https://doi.org/10.1534/genetics.112.145284

Schuenemann, R., \& Oechtering, G. (2014). Inside the brachycephalic nose: conchal regrowth and mucosal contact points after laser-assisted turbinectomy. Journal of the American Animal Hospital Association, 50(4), 237-246.https://doi.org/10.5326/JAAHA-MS-6086

Seneviratne, M., Kaye, B. M., \& Ter Haar, G. (2020). Prognostic indicators of short-term outcome in dogs undergoing surgery for brachycephalic obstructive airway syndrome. The Veterinary record, 187(10), 403. https://doi.org/10.1136/vr.105624

Shales, C. (2014). Factors, diagnosis and treatment of BOAS in dogs. Vet. Times, (11).

Shaver, S. L., Barbur, L. A., Jimenez, D. A., Brainard, B. M., Cornell, K. K., Radlinsky, M. G., \& Schmiedt, C. W. (2017). Evaluation of Gastroesophageal Reflux in Anesthetized Dogs with Brachycephalic Syndrome. Journal of the American Animal Hospital Association, 53(1), 24-31. https://doi.org/10.5326/JAAHA-MS-6281

Sławuta, P., Nicpoń, J., \& Domańska, S. (2011). Influence of the wing-of-the-nostrils correction procedure on the change of the acid-base balance parameters and oxygen concentration in the arterial blood in French bulldogs. Polish journal of veterinary sciences, 14(1), 77-80. https://doi.org/10.2478/v10181-011$0011-1$

Sławuta, P., Glińska-Suchocka, K., \& Cekiera, A. (2015). The use of elements of the Stewart model (Strong Ion Approach) for the diagnostics of respiratory acidosis on the basis of the calculation of a value of a modified anion gap (AGm) in brachycephalic dogs. Polish journal of veterinary sciences, 18(1), 217222. https://doi.org/10.1515/pjvs-2015-0027

Tappin, S. W. (2016). Canine tracheal collapse. Journal of Small Animal Practice, 57(1), 9-17. https://doi.org/10.1111/jsap.12436

Torrez, C. V., \& Hunt, G. B. (2006). Results of surgical correction of abnormalities associated with brachycephalic airway obstruction syndrome in dogs in Australia. The Journal of small animal practice, 47(3), 150-154. https://doi.org/10.1111/j.1748-5827.2006.00059.x

Trappler, M., \& Moore, K. (2011). Canine brachycephalic airway syndrome: surgical management. Compendium (Yardley, PA), 33(5), E1-E8.

Vilaplana Grosso, F., Haar, G. T., \& Boroffka, S. A. (2015). Gender, weight, and age effects on prevalence of caudal aberrant nasal turbinates in clinically healthy english bulldogs: a computed tomographic study and classification. Veterinary radiology \& ultrasound: the official journal of the American College of Veterinary Radiology and the International Veterinary Radiology Association, 56(5), 486-493. https://doi.org/10.1111/vru.12249

Winter, R. L., Saunders, A. B., Gordon, S. G., Miller, M. W., Fosgate, G. T., Suchodolski, J. S., \& Steiner, J. M. (2017). Biologic variability of cardiac troponin I in healthy dogs and dogs with different stages of myxomatous mitral valve disease using standard and high-sensitivity immunoassays. Veterinary clinical pathology, 46(2), 299-307. https://doi.org/10.1111/vcp.12495

Yoon, H., Yu, J., An, G., Bang, S., Kwon, D., Kim, H., Lee, H., Chang, J. \& Chang, D. (2020). CT and radiographic evaluation of bronchial collapsibility at forced expiration in asymptomatic brachycephalic dogs. Veterinary Radiology \& Ultrasound, 61(2), 167-180.https://doi.org/10.1111/vru.12829 